\title{
Álcool e Direção em Universitários, Comunicação Persuasiva e Prevenção
}

Persuasive Communication and Prevention in the Use of Alcohol and Driving Comunicación Persuasiva y la Prevención en el Consume de Alcohol y Conducciónn

Nemésio Dario Almeida

Doutor em Psicologia, Analista Judiciário, Psicólogo do TJ-PE

Antonio Roazzi

D.Phil Oxford, Professor Titular,

Depto Psicologia e PPG em

Psicologia Cognitiva

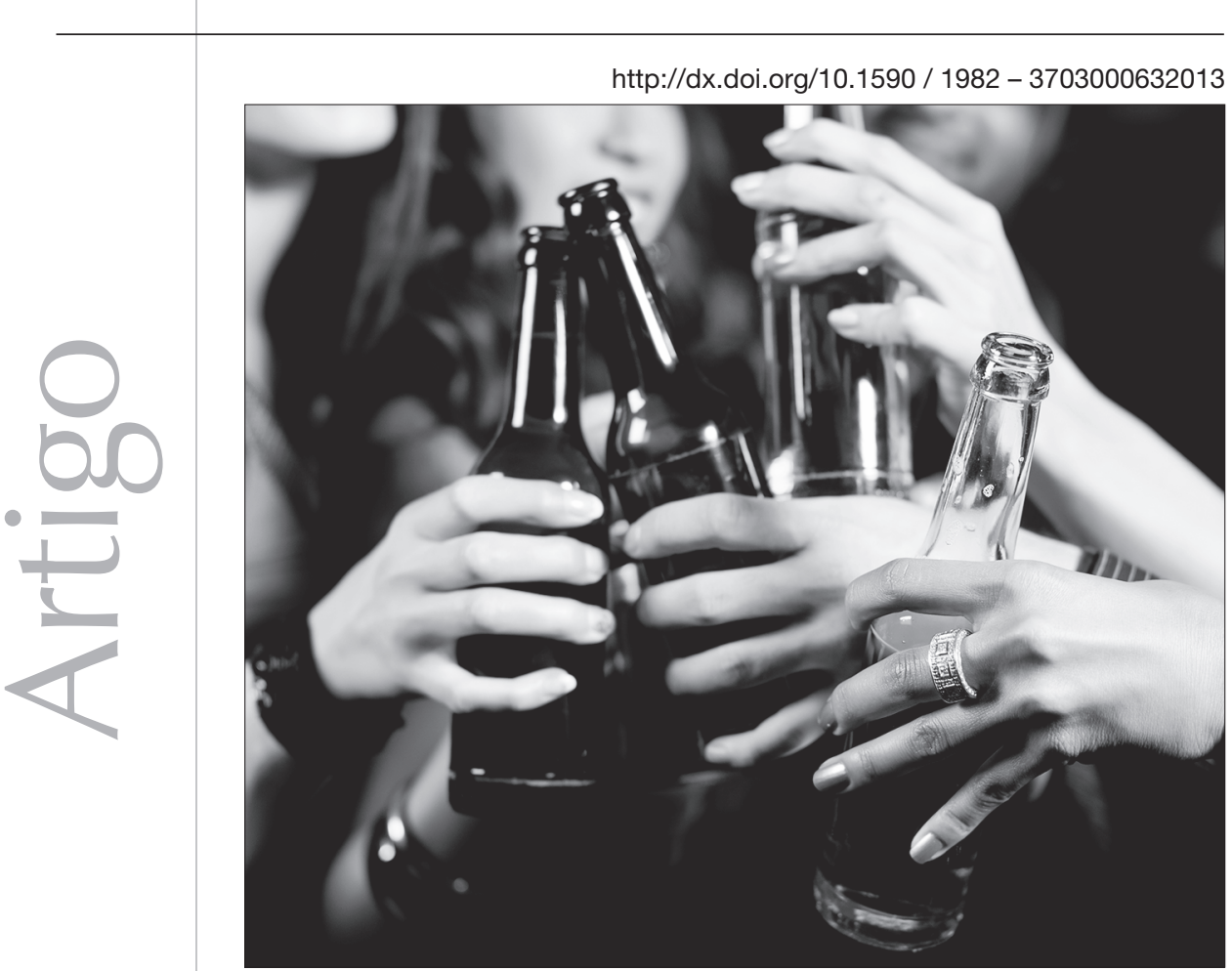


Resumo: O presente estudo teve dois objetivos a pesquisar, através da Teoria da Ação Racional (TAR): (1) testar a adequação da TAR no que diz respeito à predição da intenção para adotar o comportamento do não uso de bebidas alcoólicas e direção em estudantes universitários; (2) testar a influência de três tipos de comunicações persuasivas (positiva, negativa e irrelevante), baseadas também na TAR, sobre a intenção de adotar ainda o mesmo comportamento preventivo citado. Para tanto, considerou-se uma amostra de 163 universitários, sendo 99 mulheres e 64 homens, com 18 a 42 anos de idade, do campus UFPE. Os resultados demonstraram que, segundo a TAR, existiu uma rendição para os grupos experimentais; consideráveis percentuais de variância da variável dependente explicados pelas independentes dos grupos experimentais e controle-placebo; correlações satisfatórias e significativas para variáveis. Coube à estratégia persuasiva negativa maior variância da variável dependente. Correlações confirmaram validade teórica e metodológica à TAR. Evidenciaram-se diferentes fatores influenciando o comportamento estudado, apontando possíveis caminhos para o planejamento de programas, cujo objetivo seja estimular o não uso de álcool e direção, e estratégias preventivas, bem como congregar novos modelos (mídia, por exemplo) que elucidam com maior realismo a complexidade desse comportamento nessa significativa população.

Palavras-chave: Álcool etílico. Trânsito(Transgressões). Atitudes. Prevenção.

Abstract: The present study had two goals to research, through Theory of Reasoned Action (TRA): (1) testing the adequacy of TRA with regards to predicting intent to adopt behavior of non-use of alcoholic beverages and driving in university students; (2) testing the influence of three types of persuasive communication (positive, negative and irrelevant), based also on TRA, about the intention of adopting the aforementioned preventive behavior. For such, we considered a sample of 163 university students, 99 women and 64 men, with 18 to 42 yielding to experimental groups; considerable percentage variance of the dependent variable explained by the independent of the experimental and control-placebo groups; satisfactory and significant correlations for variables. The largest variance happened with the negative persuasive strategy of the dependent variable. Theoretical and methodological validity confirmed correlations to TRA. It became evident that different factors influenced the studied behavior, pointing out possible paths for planning programs whose aim is to stimulate no drinking and driving, and preventive strategies, as well as to bring new models (media, for example) to elucidate with greater realism the complexity of this behavior in this large population.

Keywords: Alcohol. Traffic violations. Attitudes. Prevention.

Resumen:El presente estudio tuvo dos objetivos a pesquisar, a través de la Teoría de la Acción Racional (TAR): (1) comprobar la adecuación de la TAR en lo que dice respecto a la predicción de la intención para adoptar el comportamiento de no consumir bebidas alcohólicas y la conducción en estudiantes universitarios; (2) comprobar la influencia de tres tipos de comunicaciones persuasivas (positiva, negativa e irrelevante), basadas también en la TAR, sobre la intención de adoptar todavía el mismo comportamiento preventivo citado. Para tanto, se consideró una muestra de 163 universitarios, siendo 99 mujeres y 64 hombres, con entre 18 y 42 años de edad, del campus UFPE. Los resultados demostraron que, según la TAR, existió una rendición para los grupos experimentales; considerables porcentuales de variancia de la variable dependiente explicados por las independientes de los grupos experimentales y control-placebo; correlaciones satisfactorias y significativas para variables. Cupo a la estrategia persuasiva negativa mayor variancia de la variable dependiente. Correlaciones confirmaron validez teórica y metodológica a la TAR. Se evidenciaron diferentes factores influenciando el comportamiento estudiado, apuntando posibles caminos para la planificación de programas, cuyo objetivo sea estimular o no el consumo de alcohol y conducción, y estrategias preventivas, así como congregar nuevos modelos (medios, por ejemplo) que elucidan con mayor realismo la complejidad de ese comportamiento en esa significativa población.

Palabras clave: Etanol. Tránsito (Transgresores). Actitudes. Prevención. 
O tema relacionado aos acidentes de trânsito ocasionados por motoristas alcoolizados COmeça a ter uma dimensão mais ampla na literatura psicológica. De um lado, embora sejam realizados programas de prevenção e intervenção quanto a não dirigir sob o efeito do álcool continuamente, pesquisas demonstram que os índices de acidentes de trânsitos provocados por motoristas alcoolizados ainda são considerados altos em todo o mundo (Almeida, 2010a; Duailibi, Pinsky, \& Laranjeira, 2010; Thielen \& Soares, 2009).s brasileiros estão morrendo mais em acidentes com transporte terrestre(ATTs), é o que aponta o Sistema de Informações de Mortalidade (SIM) do Ministério da Saúde (2012), cujos dados de 2011 revelam: no total de 153.542 internações por ATTs ocorridas no Sistema Único de Saúde (SUS) em 2011, os homens representaram $78,4 \%$ das internações e as muIheres, $21,6 \%$. A taxa de internações hospitalares por essa causa foi de 8,0 por $10 \mathrm{mil}$ habitantes, variando de 12,8 por 10 mil homens a 3,8 por 10 mil mulheres, sendo essa taxa de internação, entre os homens, 3,3 vezes a estimada entre as mulheres.

Para a faixa etária de 20 a 39 anos, a maior taxa de internações por ATTs foi de 19,8 por 10 mil homens e, para mulheres, foi de 5,0 por 10 mil mulheres. A faixa etária de 20 a 59 anos foi a de maior proporção das hospitalizações por ATTs (71,3\%), seguindo-se o grupo de 10 a 19 anos de idade (7,0\%). A maior proporção de internações por ATTs foi de motociclistas $(50,5 \%)$, com as taxas mais elevadas, principalmente, para os homens (6,9 por 10 mil homens), o que representa uma taxa 5,5 vezes maior que a das mulheres (1,3 por 10 mil mulheres) (Ministério da Saúde, 2012).

Ainda de acordo com o SIM do Ministério da Saúde (2012), em relação aos ATTs, foi observado um incremento de $227 \%$ nas taxas de internações com vítimas motociclistas entre 2002 e 2011. Esse expressivo aumento pode estar relacionado ao aumento de motocicletas e motociclistas. Também é importante lembrar que os motociclistas são extremante vulneráveis aos ATTs, representando a maior proporção de internações por ATTs $(50,5 \%)$, o que também foi observado por outros estudos (Mendes et al., 2012). Tem sido descrito que, para os motociclistas, o uso do capacete pode reduzir em $29 \%$ o risco de lesões fatais e em $67 \%$ o risco de traumatismo cranioencefálico.

Os números revelam que o país vive uma verdadeira epidemia de lesões e mortes no trânsito, alerta o próprio Ministério da Saúde, observa-se ainda que a World Health Organization (WHO)aponta que o Brasil ocupa o quinto lugar em ocorrências como essas; estamos atrás apenas da Índia, China, EUA e Rússia (WHO, 2011). Diversos estudos alertam para o comportamento no trânsito de forma a apoiar uma fiscalização mais rigorosa e intervenções educativas na prevenção dos acidentes de trânsito (Almeida, 2003, 2006, 2010b, 2011, no prelo; Duailibi, Pinsky, \& Laranjeira, 2010; Maia, 2009; Tawil, 2007). Sendo assim, a Teoria da Ação Racional(TAR) de Ajzen e Fishbein (1980) e Fishbein e Ajzen (1975) é um dos modelos que tem sido empregado para descrever, explicar, predizer e prescrever comportamentos volitivos de prevenção de doenças e de promoção da saúde (Ajzen, Albarracín,\& Hornik, 2007; Almeida et al., 2004; Bastos, 2000; Bezerra, 1997; Breinbauer \& Maddaleno, 2008a, 2008b;Cavalcanti et al., 2007; Cunha \& Dias, 2008; D'Amorim, 2004; Dias, 1995; Dias \& Cavalcanti, 2005; Fishbein \& Ajzen, 2010;Formiga, 2002; Formiga, Dias,\& Saldanha, 2005; Guimarães, 2004; Michener, Delamate,\&Myers, 2005;Moutinho, 2000; Moutinho \& Roazzi, 2010; Santanna, 2005).

De acordo com o modelo, o comportamento da pessoa é determinado pela sua intenção comportamental de realizar esse comportamento. Dois fatores principais determinam a intenção comportamental do indivíduo: um fator pessoal (sua atitude) e um fator social (sua norma subjetiva). Cada um desses fatores é também uma função de dois outros determinantes. A atitude da pessoa é uma função daquilo que ela crê que irá acontecer ao desempenhar o comportamento (suas crenças comportamentais) e das avaliações que ela faz a respeito das consequências da execução desse mesmo comportamento (suas avaliações das consequências). O segundo componente, norma subjetiva, é uma função 
daquilo que a pessoa acredita que os outros acham que ela deve fazer (suas crenças normativas) e da motivação para corresponder ao que os outros acham que ela deve fazer (suas motivações para concordar) (D'Amorim, 1996; D'Amorim\& Vera, 1996; Dela Coleta, 2004).

No Brasil, em que pese à dificuldade de reduzir os números de acidentes provocados por motoristas alcoolizados, poucas pesquisas têm examinado o papel, em conjunto, da atitude, norma e intenção como fatores determinantes desse comportamento (Almeida, 2010a; Almeida et al., 2004; Almeida \& Roazzi, no prelo). Análises conjugadas dos processos empíricos relacionados aos contextos pessoais, sociais e cognitivos de não ingerir álcool e direção também se mostram escassas (Almeida, 2010a; Almeida et al., 2004).

Levando em conta tais considerações e utilizando a TAR, Almeida (2010) levou a cabo uma investigação com 488 estudantes universitários com o objetivo de testar a aplicabilidade do modelo na explicação e predição da "intenção comportamental de não ingerir álcool e dirigir". Em um primeiro momento, o autor realizou o levantamento das crenças sobre o comportamento de ingerir álcool e direção e identificou aquelas crenças modais salientes, crenças eliciadas que corresponderam a uma frequência maior ou igual a $75 \%$ do total de crenças emitidas pela amostra. Em um segundo momento, verificou que as variáveis preditoras crenças normativas, a atitude e as crenças comportamentais explicaram, conjuntamente, $22 \%$ da variância da variável critério, intenção comportamental.

De posse dos resultados obtidos por Almeida (2010a) e (1) considerando as metas e os princípios expressos pela Política Nacional do governo federal que dispõem sobre a inibição do consumo de bebida alcoólica por condutor de veículos automotor, e dá outras providências (Brasil, 2008), considerando, também, o processo de subjetividade do consumo de álcool e outras drogas e as políticas públicas brasileiras pelo Conselho Federal de Psicologia (2010); (2) considerando, ainda, os impactos sociais e econômicos dos acidentes de trânsito nas aglomerações urbanas e rodovias brasileiras pelo Instituto de Pesquisa Econômica Aplicada-Ipea (2003, 2006); (3) considerando, ao mesmo tempo, que uma das formas mais eficientes de reverter esse quadro seriam campanhas de comunicação em massa, incentivando a prevenção do comportamento de não ingerir bebidas alcoólicas quando for dirigir e; (4) considerando, por fim, que o modo de diligenciar tais mudanças comportamentais na população-alvo em prol da prática do não dirigir sobre o efeito de álcool seria elaborar e testar modelos de comunicações persuasivas alicerçadas nos fatores preditores da "intenção comportamental de não ingerir álcool e dirigir" identificados por Almeida (2010a), é que foi planejada e executada a pesquisa em questão, com os objetivos e hipóteses empíricas associadas elencadas adiante.

\section{Objetivos e hipóteses}

Dessa forma, nesta seção e na subsequente, foram ressaltadas as normas prescritas por Günther (2003) e Ramos-Álvarez e Catena (2004) no que se refere à (1) adequabilidade e estrutura causal das hipóteses, (2) adequabilidade do desenho experimental, (3) operacionalização das variáveis dependentes e independentes, (4) validade e confiabilidade do instrumento de coleta de dados, (5) seleção da amostra e (6) condução ética da investigação.

Optou-se, também, pelo uso do termo yielding (Ajzen \& Fishbein, 1980; Almeida, 2010a; Dias, 1995; Fishbein \& Ajzen, 1975) no original, para caracterizar com precisão o efeito de rendição/capitulação dos participantes às mensagens persuasivas (variáveis independentes) sobre a adoção do comportamento investigado, a mudança da magnitude de suas intenções comportamentais (variável dependente) em função a não ingerir álcool e direção.

\section{Objetivo geral}

Verificar experimentalmente os efeitos de impacto das mensagens persuasivas na variável 
dependente intenção comportamental do não ingerir álcool e direção.

\section{Hipótese geral}

O escore médio da variável dependente "intenção comportamental do não ingerir álcool e direção" é significativamente maior para o Grupo Experimental 1-GE1, Grupo Experimental 2-GE2, controle-placebo-GCP e somente-controle-GSC.

\section{Objetivos específicos}

Verificar a influência de três tipos de mensagens persuasivas - nas versões positiva, negativa e irrelevante - sobre a variância da variável dependente intenção comportamental do não ingerir álcool e direção, por parte das variáveis independentes. Testar a adequabilidade da Teoria da Ação Racional na predição da variável critério na amostra e comportamento estudados.

\section{Hipótese específica 1}

A comunicação persuasiva positiva-Grupo Experimental 1-GE1 tem maior influência na explicação da variância da intenção comportamental não ingerir álcool e direção pelas variáveis preditivas, que a comunicação persuasiva negativa-Grupo Experimental 2-GE2. Hipótese específica 2

A comunicação persuasiva positiva-Grupo Experimental 1-GE1 tem maior impacto na explicação da variância da intenção comportamental do não ingerir álcool e direção pelas variáveis independentes, que a comunicação persuasiva irrelevante-Grupo Controle Placebo-GCP.

\section{Hipótese específica 3}

A comunicação persuasiva negativa-Grupo Experimental 2-GE2 tem maior impacto na explicação da variância da intenção com- portamental do não ingerir álcool e direção pelas variáveis antecedentes, que a comunicação persuasiva irrelevante-Grupo Controle Placebo-GCP.

\section{Hipótese específica 4}

A Teoria da Ação Racional representa um modelo ajustado adequadamente na descrição, explicação, predição e prescrição da intenção comportamental do não ingerir álcool e direção em universitários.

\section{Método}

O método adotado seguiu as prescrições dos autores da Teoria da Ação Racional (Ajzen \& Fishbein, 1980; Fishbein \& Ajzen, 1975), bem como aquelas próprias para estudos de natureza experimental (Ramos-Álvarez \& Catena, 2004). O projeto foi aprovado pelo Comitê de Ética do Centro de Ciências da Saúde da Universidade Federal de Pernambuco (UFPE), sob Registro CEP/CCS/UFPE № 167/08, em 19/08/2008.

\section{Etapa I: Elaboração das comuni- cações persuasivas}

A construção das comunicações persuasivas seguiu as normas do modelo da Teoria da Ação Racional (Ajzen \& Fishbein, 1980; Fishbein \& Ajzen, 1975), sendo estas elaboradas nas versões positiva, negativa e irrelevante (Almeida, 2010a). As duas primeiras comunicações tiveram por objetivo influenciar os componentes atitudinal, normativo de não ingerir álcool e direção, sendo as crenças selecionadas para a elaboração de ambas, aquelas crenças modais salientes que se mostraram mais discriminativas, no estudo de Almeida (2010a), como as crenças de "provocar acidentes", "risco de vida", "reduzir atenção", "reduzir reflexos", "sonolência", entre os grupos de respondentes que intencionavam e não intencionavam realizar o comportamento investigado. A comunicação positiva exortava as vantagens em se adotar 
o comportamento de não ingerir álcool e direção; a negativa, as desvantagens em não se adotar o comportamento de não ingerir álcool e direção.

\section{Etapa II: aplicação e estudo experimental das comunicações persuasivas}

\section{População e amostra}

As informações obtidas referentes aos dados sociodemográficos dos participantes, com respectivos frequência e percentual de ocorrência, estão ilustrados na Tabela 1.A amostra foi composta de 163 estudantes universitários da UFPE, sendo $60,7 \%$ do gênero feminino e 39,3\% do gênero masculino, com idade que variou entre 18 e mais de 42 anos, pertencentes a diferentes cursos da UFPE. Observa-se que a média de idade encontrada foi de 25,4 anos $(D P=7,02)$, em relação ao estado civil, $85,2 \%$ são solteiros e 14,7\% referiram-se ser casado e separado. A religião católica foi a predominante, tendo sido citada por $45,3 \%$ dos participantes, enquanto os evangélicos referiram-se a 20,2\%. Dentre os cursos de maior figuração na amostra, encontram-se Ciências Contábeis (25,8\%), Terapia Ocupacional (24,5\%) e Educação Física (19,6\%).

Tabela 1.

Estatística Descritiva das Variáveis Sociodemográficas em termos reais e percentuais $(n=163)$. Recife (PE), 2009-2010.

\begin{tabular}{|c|c|c|c|c|}
\hline Sexo & $\mathrm{F}$ & $\%$. & f vál. & \% cum. \\
\hline Feminino & 99 & 60.7 & 60.7 & 60.7 \\
\hline Masculino & 64 & 39.3 & 39.3 & 100.0 \\
\hline Total & 163 & 100 & 100 & 100 \\
\hline Idade & $\mathrm{F}$ & $\%$. & f vál. & $\%$ cum. \\
\hline Entre 18 e 23 anos & 83 & 50.9 & 50.9 & 50.9 \\
\hline Entre 24 e 29 anos & 53 & 32.5 & 32.5 & 83.4 \\
\hline Entre 30 e 41 anos & 18 & 11.0 & 11.0 & 94.4 \\
\hline Mais de 42 anos & 9 & 5.5 & 5.5 & 100.0 \\
\hline Total & 163 & 100 & 100 & 100 \\
\hline Estado Civil & $\mathrm{F}$ & $\%$. & f vál. & \% cum. \\
\hline Solteiro & 139 & 85.2 & 85.2 & 85.2 \\
\hline Casado/Separado & 24 & 14.7 & 14.7 & 100.0 \\
\hline Total & 163 & 100 & 100 & 100 \\
\hline Religião & $\mathrm{F}$ & $\%$. & f vál. & $\%$ cum. \\
\hline Católica & 74 & 45.3 & 62.1 & 62.1 \\
\hline Evangélica & 33 & 20.2 & 27.7 & 89.8 \\
\hline Espírita & 11 & 6.7 & 9.2 & 99.0 \\
\hline Ateu & 1 & 0.6 & 0.8 & 100.0 \\
\hline Não-relatadas & 44 & 26.9 & - & - \\
\hline Total & 163 & 100 & 100 & 100 \\
\hline Cursos Universitários & $\mathrm{F}$ & $\%$. & f vál. & $\%$ cum. \\
\hline Ciências Contábeis & 42 & 25.8 & 25.8 & 25.8 \\
\hline Terapia Ocupacional & 40 & 24.5 & 24.5 & 50.3 \\
\hline Educação Física & 32 & 19.6 & 19.6 & 69.9 \\
\hline História & 18 & 11.0 & 11.0 & 80.9 \\
\hline Enfermagem & 15 & 9.2 & 9.2 & 90.1 \\
\hline Letras & 5 & 3.1 & 3.1 & 93.2 \\
\hline Outros & 11 & 6.7 & 6.7 & 100.0 \\
\hline Total & 163 & 100 & 100 & 100 \\
\hline
\end{tabular}




\section{Desenho experimental}

Foi composto por um planejamento poligrupal (ampliação do delineamento $\mathrm{n}-6$ de Campbell \& Stanley, 1979), com distribuição equânime dos participantes para as condições comunicação persuasiva positiva (grupo experimental 1-GE1 = 42 participantes), comunicação persuasiva negativa (grupo experimental 2-GE2 $=41$ participantes), (comunicação persuasiva irrelevante (grupo controle-placebo - GCP = 40 participantes) e sem comunicação persuasiva (grupo somente-controle - GSC $=40$ participantes). A escolha desse desenho atendeu a critérios de controle das principais fontes de invalidação metodológica interna e externa (Campbell \& Stanley, 1979).

\section{Descrição das variáveis depen- dente e explicativa}

Ajzen e Fishbein (1980) e Fishbein e Ajzen (1975) criaram um questionário como instrumento de mensuração para um ato único dos diversos construtos do modelo teórico. A primeira decisão, de grande importância, é a escolha do comportamento específico a ser estudado na população-alvo, como, por exemplo, escolhemos o não uso de álcool e direção por estudantes universitários.

De acordo com os autores da TAR, a construção do instrumento passa por diversas etapas, assim, na primeira, uma amostra de participantes, com as mesmas características dos futuros respondentes, fornece, por meio de entrevista, uma lista das vantagens e desvantagens, como, por exemplo, o uso de álcool e direção por universitários. A pergunta é feita em caráter pessoal, não se tratando do comportamento geral do uso de álcool e direção, mas sim de uma ação da pessoa. As respostas irão constituir as crenças comportamentais (CC) do grupo, já que é uma listagem das possíveis consequências do comportamento em questão.

Além dessa pergunta, os respondentes devem listar as pessoas cuja opinião acerca do comportamento do uso de álcool e direção seja importante para elas, essas pessoas, chamadas de referentes, embasam as crenças normativas do grupo acerca da aceitação social do comportamento estudado. Também o questionário contém perguntas referentes a dados sociodemográficos dos participantes, tais como sexo, idade, entre outras.

O número de respondentes a serem entrevistados é determinado empiricamente, por meio de, no mínimo, cinco entrevistas seguidas, nas quais as respostas obtidas nada acrescentem às vantagens e desvantagens do comportamento e ao número dos referentes.

Os dados obtidos passaram por uma análise de conteúdo que procurou agrupar as respostas com o mesmo significado, dadas sob forma diferente; as mesmas vantagens ou desvantagens podem ser expressas de modo diverso. Após a análise, será calculada a frequência de cada tipo de resposta, sendo as mais frequentes consideradas como crenças modais salientes. Essas crenças vão constituir os itens do instrumento de pesquisa, sendo necessário um mínimo de aceitação por 75\% dos membros do grupo entrevistado.

Em uma segunda etapa, o instrumento é elaborado sob a forma de questionário, utilizando escalas de 7 pontos que vão do tipo provável (7) a improvável (1) para a maioria das variáveis: intenção (I), crenças comportamentais (CC) e normativas $(\mathrm{CN})$, motivação para concordar com a opinião dos referentes (M) e norma subjetiva (NS).

A avaliação das crenças é respondida em uma escala de prudente (7) a imprudente (1) e a atitude, por meio de um diferencial semântico cujas dimensões variam com o comportamento estudado.

Sendo assim, ao se investigar o comportamento do uso de álcool e direção, com universitários, obtivemos quatro crenças modais salientes e três referentes. Dessa forma e de acordo com D'Amorim (1996), as crenças obtidas são mensuradas duas vezes - quanto à possibilidade das consequências e quanto à sua avaliação.

Os referentes também figuram duas vezes no questionário - como fontes de opinião e como objeto da motivação para acatar essa 
opinião. Conforme Ajzen e Fishbein (1980) e Fishbein e Ajzen (1975), as questões do instrumento obedeceram a uma ordem determinada de apresentação, como se segue: Atitude (AT) - mensuração direta - um único item constituído pela soma das respostas obtidas nas várias dimensões de um diferencial semântico; As Crenças Comportamentais (CC) - as que foram consideradas crenças modais salientes na primeira etapa; A Avaliação das Crenças (AC); A Norma Subjetiva (NS) - que é uma mensuração direta, e por fim, a Motivação para Concordar com os referenciais (M).

\section{Instrumento}

O instrumento utilizado para a mensuração dos efeitos das comunicações persuasivas sobre a variável critério foi aquele elaborado e validado por Almeida (2010a), o Comportamento de Saúde dos Estudantes Universitários de Recife, conforme prescrições da TAR - de formato estruturado e respostas a "lápis e papel" -, composto por 27 itens na forma de escalas bipolares - de diferencial semântico (útil-inútil, prudente-imprudente) e de probabilidade subjetiva (provável-improvável) - ancorada em um continuum de sete pontos para a medida de todas as variáveis do modelo. A apresentação e conclusão do instrumento seguiram as orientações de Günther (2003).

Ainda sobre o instrumento utilizado, as perguntas foram elaboradas a partir das crenças modais salientes, sendo que o questionário procurou medir as atitudes dos respondentes em relação aos comportamentos preventivos, suas avaliações das consequências desses comportamentos, a intensidade das suas crenças comportamentais e normativas, como também a norma subjetiva, as crenças normativas e as motivações para concordar com os referentes (Almeida, 2010a).

Dessa forma, temos, no que se refere aos elementos constituintes da TAR, que a elaboração das comunicações persuasivas para o estudo do não ingerir álcool e direção contemplava os critérios metodológicos de Ajzen e Fishbein (1980) e Fishbein e Ajzen (1975).

\section{Procedimento}

A amostra foi de conveniência (Cozby, 2009), como estratégia desenvolvida no momento da aplicação, o grupo somente-controle GSC foi o primeiro contatado, vindo a seguir as condições controle-placebo - GCP, experimental 2 - GE2 e experimental 1 - GE1, nessa ordem, com o fito de se evitar a contaminação do efeito de conteúdo das comunicações persuasivas entre os participantes (Campbell \& Stanley, 1979).

Inicialmente, foram feitos contatos com os professores de disciplinas ministradas nas diversas áreas acadêmicas do Campus da Universidade Federal de Pernambuco. Foi-lhes explicado que a pesquisa teria por objetivo conhecer aspectos relacionados ao comportamento de universitários frente ao uso de bebidas alcoólicas e direção.

Realizada a apresentação, o pesquisador distribuiu o material da pesquisa e procedeu à leitura das instruções impressas neste. Após o esclarecimento de algumas dúvidas, os participantes responderam o instrumento apresentado. Nenhum aluno(a) se recusou a participar do estudo e o tempo médio de leitura das comunicações e preenchimento do questionário variou entre 15 a 25 minutos aproximadamente.

\section{Resultados}

Foram utilizados para os tabelamentos e análises estatísticas dos resultados apresentados a seguir o software do Pacote Estatístico para Ciências Sociais - SPSSwin (Statistical Package of the Social Sciences for Windows), versão 15.0 como editor estatístico (Bisquerra, Sarriera,\& Martínez, 2007), por meio do qual também foram eliminados aqueles respondentes que apresentaram altos índices (cerca de $1 / 3$ ) de missing values (valoresomissos) para o total de itens do instrumento, bem como os que se apresentaram como outliers (respostas com valores extremos).

Análises das matrizes de correlações $r$ de Bravais-Pearson foram realizadas com a fi- 
nalidade de descrever os tipos de relações existentes entre as medidas diretas e indiretas dos componentes normativos e atitudinais, outra finalidade foi a de verificar as relações desses componentes com a medida da variável dependente: a intenção comportamental. Análises de regressões lineares múltiplas também foram realizadas para determinar o percentual de variância, devido à influência das diferentes condições experimentais nas variáveis independentes do modelo (Atitude, Norma Subjetiva, Crença Comportamental e Crença Normativa) e de seus impactos na variável dependente Intenção Comportamental.

Também uma análise de variância unidirecional (one-way ANOVA) foi efetuada entre as médias da variável intenção comportamental nos grupos experimentais e a média da mesma variável no grupo controle. $\mathrm{O}$ cálculo da análise de variância teve a finalidade de verificar a existência de efeitos de capitulação (yielding), decorrentes dos tipos de comunicações veiculadas, para a variável de interesse, a intenção comportamental.

Finalmente, com o objetivo de estudar o efeito de alguma fonte externa responsável pelos resultados contraditórios - quanto em adotar o comportamento de não ingerir bebidas alcoólicas e direção, foram efetuadas análises do teste qui-quadrado, comparando os participantes da pesquisa em função das quatro condições experimentais.

\section{Análises das matrizes de corre- lações $r$ de Pearson das variáveis da TAR}

Feitas essas ressalvas, um primeiro passo para identificar os determinantes da intenção comportamental está em precisar a força e a direção do relacionamento entre as variáveis envolvidas. As medidas de correlação são importantes justamente por permitirem esse tipo de informação (Tabela 2).

Para a condição de tratamento Comunicação Persuasiva Positiva, o resultado das correlações em relação à intenção comportamental quanto em adotar o comportamento de não ingerir bebidas alcoólicas e direção entre as variáveis da TAR, foram consideradas: medida direta da atitude, crença comportamental geral, crença normativa geral e medida direta da norma subjetiva.

Dentre as variáveis correlacionadas com a intenção - quanto em adotar o comportamento de não ingerir bebidas alcoólicas e direção, a crença comportamental geral obteve maior coeficiente de correlação, seguida da variável atitude e da variável a crença normativa geral. Nota-se, ainda, dessa forma, que correlação moderada e positiva foi encontrada entre as variáveis crenças comportamentais gerais e atitude, tal como previsto pela TAR. A correlação mais baixa com a intenção comportamental foi a variável norma subjetiva. 
Esses resultados sugerem que as variáveis: crença comportamental geral e atitude são os principais determinantes da intenção comportamental - quanto em adotar o comportamento de não ingerir bebidas alcoólicas e direção.

\section{Tabela 2}

Matriz de correlações $r$ de Pearson entre as variáveis da Teoria da Ação Racional $(n=163)$. Recife (PE), 2009-2010.

\begin{tabular}{|c|c|c|c|c|c|}
\hline Grupos & Variáveis & AT & CCG & NS & CNG \\
\hline Grupo & IC & $0,377^{\star}$ & $0,582^{\star \star}$ & $0,347^{\star}$ & 0,163n.s. \\
\hline \multirow[t]{4}{*}{ Experimental 1} & AT & - & $0,626^{\star \star}$ & $0,376^{*}$ & $0,350^{\star}$ \\
\hline & CCG & $0,626^{\star \star}$ & - & $0,571^{\star \star}$ & $0,383^{*}$ \\
\hline & CNG & $0,376^{*}$ & $0,571^{\star \star}$ & - & 0,233n.s. \\
\hline & NS & $0,350^{\star}$ & $0,383^{*}$ & 0,233 n.s. & - \\
\hline Grupo & $\mathrm{IC}$ & 0,182 n.s. & 0,273 n.s. & $0,644^{\star \star}$ & 0,093 n.s. \\
\hline \multirow[t]{4}{*}{ Experimental 2} & AT & - & 0,246 n.s. & 0,031 n.s. & 0,149 n.s. \\
\hline & CCG & 0,246 n.s. & - & 0,007 n.s. & -0,034 n.s. \\
\hline & CNG & 0,031 n.s. & 0,007 n.s. & - & $0,434^{\star \star}$ \\
\hline & NS & 0,149 n.s. & -0,034 n.s. & $0,434^{\star \star}$ & - \\
\hline Grupo controle & IC & $0,425^{\star \star}$ & $0,541^{\star \star}$ & $0,405^{\star \star}$ & $0,337^{\star}$ \\
\hline \multirow[t]{4}{*}{ Placebo } & AT & - & $0,728^{\star \star}$ & $0,450^{\star \star}$ & $0,385^{\star}$ \\
\hline & CCG & $0,728^{\star \star}$ & - & $0,625^{\star \star}$ & $0,530^{\star \star}$ \\
\hline & CNG & $0,450^{\star \star}$ & $0,625^{\star \star}$ & - & $0,626^{\star \star}$ \\
\hline & NS & $0,385^{\star}$ & $0,530^{\star \star}$ & $0,626^{\star \star}$ & - \\
\hline Grupo somente & IC & 0,070 n.s. & 0,158 n.s. & 0,041 n.s. & 0,103 n.s. \\
\hline \multirow[t]{4}{*}{ Controle } & AT & - & $0,316^{*}$ & $-0,222$ n.s. & 0,112 n.s. \\
\hline & CCG & $0,316^{\star}$ & - & $0,548^{\star \star}$ & $0,497^{\star \star}$ \\
\hline & CNG & 0,222 n.s. & $0,548^{\star \star}$ & - & 0,162 n.s. \\
\hline & NS & 0,112 n.s. & $0,497^{\star \star}$ & 0,162 n.s. & - \\
\hline
\end{tabular}

n.s.: não significativa; IC: intenção comportamental (variável dependente); AT: atitude (variável independente); CCG: crença comportamental geral (variável independente); CNG: crença normativa geral (variável independente); NS: norma subjetiva (variável independente).

${ }^{*} p<0,05$;

$* * 0,01$.

\section{Análises de regressões lineares múltiplas}

A partir da Tabela 3, exibe-se a análise de regressão (Stepwise) realizada, para as quatro condições de estudo: o Grupo Experimental 1-GE1, Grupo Experimental 2-GE2, Grupo Controle-Placebo-GCP e Grupo Somente-Controle-GSC, tendo como variável dependente a intenção comportamental - quanto em adotar o comportamento de não ingerir bebidas alcoólicas e direção, e como variáveis independentes a crença comportamental geral e a crença normativa geral. As medidas diretas da Atitude e da Norma Subjetiva não compuseram a equação de regressão nestas condições experimentais. 
O resultado apresentado pelo GE1, em relação à intenção - quanto em adotar o comportamento de não ingerir bebidas alcoólicas e direção, tem-se que a única variável preditora foram as crenças comportamentais gerais, explicando 33,9\% da variância dessa intenção. Contudo, as variáveis, atitude, norma subjetiva e crenças normativas gerais não contribuíram de forma significativa para explicação da variância do mesmo.

Para o GE2, observar-se que, quanto à intenção em adotar o comportamento de não ingerir bebidas alcoólicas e direção, as variáveis crenças normativas gerais e crenças comportamentais gerais foram indicadas como variáveis preditoras da referida intenção. As crenças normativas gerais destacam-se com maior poder preditivo, explicando $41,5 \%$ da variância da intenção quanto em adotar o comportamento de não ingerir bebidas alcoólicas e direção, frente a 7,2\% das crenças comportamentais gerais, ficando as variáveis atitude e norma subjetiva excluída da equação.

Ainda na Tabela 3, o resultado apresentado pelo Grupo Controle-Placebo (GCP - comunicação persuasiva irrelevante), em relação à intenção - quanto em adotar o comportamento de não ingerir bebidas alcoólicas e direção, tem-se que a única variável preditora foram as crenças comportamentais gerais, explicando 29,3\% da variância dessa intenção.

\section{Tabela 3}

Regressão linear múltipla (método stepwise) para a variável dependente intenção comportamental de não ingerir bebida alcoólica e direção ( $n=163)$. Recife (PE), 2009-2010.

\begin{tabular}{llllllll}
\hline Grupos & $\begin{array}{l}\text { Variáveis } \\
\text { Independentes }\end{array}$ & RM & R2 & $\begin{array}{l}\text { R2 } \\
\text { Change }\end{array}$ & F ch & Gl & $\rho$ ch \\
\hline Experimental 1 & CCG & 0,58 & 0,33 & 0,339 & 20,47 & 1,400 & 0,000 \\
Experimental 2 & CNG & 0,64 & 0,41 & 0,415 & 27,62 & 1,390 & 0,000 \\
& CCG & 0,69 & 0,48 & 0,072 & 5,33 & 1,380 & 0,026 \\
Controle-placebo & CCG & 0,54 & 0,29 & 0,293 & 15,71 & 1,380 & 0,000 \\
& & & & & & & \\
Somente- controle & CCG & 0,44 & 0,19 & 0,197 & 39,41 & 1,161 & 0,000 \\
& CNG & 0,52 & 0,27 & 0,075 & 16,45 & 1,160 & 0,000
\end{tabular}

CCG: crença comportamental geral; CNG: crença normativa geral.

\section{Análise de variância unidirecional (one-way ANOVA)}

Com o objetivo de detectar ou não efeitos de capitulação (yielding) para os GE1 e GE2 quando comparados aos GCP e GSC, foi realizada uma one-way ANOVA. Dessa forma, os resultados revelaram diferenças significativas entre as médias dos escores das três condições experimentais de comunicações persuasivas e o grupo de controle, tem-se que o GE1 apresentou um escore médio de intenção comportamental de 6,26, sendo este estatisticamente diferente quando comparado aos demais grupos experimentais e ao grupo controle $\left(F_{(3.162)}=3,560\right.$ ecorrelação significante ao nível dep < 0,016), na adoção do comportamento do não uso de bebidas e direção em universitários. Observa-se também que a média da intenção comportamental do grupo experimental 2 apresentou um escore de 6,10, já o grupo experimental 3 apresentou média de 6,05 e o escore médio do grupo controle (sem comunicação persuasiva) foi de 6,80. 


\section{Análise dos testes qui-quadrado}

Com a finalidade de se comparar os percentuais de variância da variável efeito explicados pelas variáveis antecedentes, foram realizados testes qui-quadrado entre as quatro condições de estudo.

Os resultados obtidos demonstraram que houve diferenças para as quatro condições de estudo, quando comparadas às variâncias explicadas da variável critério pelas variáveis efeitos $\left({ }^{2}(3)=12,71 ; p<0,005\right)$. Contudo, essas diferenças significativas ficaram localizadas para o GE1 e GSC $\left({ }^{2}(1)=3,725 ; p<\right.$ $0,054)$; e entre GE2 e GSC ( ${ }^{2}(1)=10,842$; $p<0,001)$; entre GCP e GSC $\left({ }^{2}(1)=10,17\right.$; $p<0,001)$, não sendo observadas diferenças significativas entre os GE1 e GE2 $\left({ }^{2}(1)=\right.$ $1,37 ; p>0,001)$, e entre GE1 e GCP $\left({ }^{2}(1)=\right.$ $1,26 ; \mathrm{p}>0,001)$, e entre GE2 e GCP $\left({ }^{2}(1)=\right.$ $0,02 ; p>0,001)$.

Temos então que os resultados obtidos por meio das comparações efetuadas permitem concluir que realmente houve um impacto efetivo decorrente do efeito experimental no grupo submetido à comunicação persuasiva positiva, negativa e irrelevante em relação ao grupo de controle, no que se refere à variável dependente, a intenção comportamental do não uso de bebidas alcoólicas e direção em universitários.

\section{Discussão}

De acordo com a Tabela 2, é possível observar que na condição comunicação persuasiva positiva foi encontrada relações significativas entre quase todas as variáveis independentes e a variável critério, a intenção comportamental, demonstrando, assim, a eficácia deste estilo de comunicação no que se refere à adoção do comportamento de não ingerir bebidas alcoólicas e direção em universitários, bem como a adequação do modelo da TAR à elaboração das mensagens persuasivas.

Conforme a Tabela 3, as análises de regressão múltipla demonstraram que, na condição comunicação negativa, as variáveis crenças comportamentais e crenças normativas, explicaram juntas $41,5 \%$ da variância total na determinação da intenção comportamental do não uso de álcool e direção. Já na condição experimental comunicação positiva, a análise de regressão demonstrou que, a variável, crenças comportamentais, explicou sozinha, 33,9\% da variância total da intenção de adotar o comportamento em questão, o que vem a demonstrar a importância dos aspectos psicossociais para o comportamento estudado. Diante do que foi exposto, o conteúdo da mensagem persuasiva negativa, assim como o da mensagem positiva, sugere também ser influente na variância da intenção comportamental, uma vez que a admoestação da mensagem negativa foi no sentido de denotar as desvantagens no consumo de álcool e direção entre os universitários.

Neste estudo, as comunicações persuasivas (nas versões positiva, negativa e irrelevante) foram elaboradas considerando os componentes que, segundo a Etapa 2 desta mesma investigação, constituem-se as crenças comportamentais e crenças normativas dessa população em relação ao não consumo etílico e direção, os quais para as crenças comportamentais foram: reduzir a atenção, os reflexos, risco de vida, sonolência, provocar acidentes, perder a consciência e envolver-se em violência e para os referentes salientes dessa mesma população os pais, irmãos, os pares e os profissionais de saúde. Considerando-se o índice de variância explicada por esses componentes, sugere-se que os programas educativos/preventivos devem abordá-los sempre que se destinarem à implementação do referido comportamento nesse estrato populacional.

Em relação aos resultados entre as médias das três condições experimentais de comunicações persuasivas e o grupo controle, observa-se que o grupo experimental 1 - GE1 mostrou um escore médio de intenção comportamental de 6,26 sendo este estatisticamente diferente quando comparado aos demais grupos experimentais e ao grupo controle $\left(F_{(3.162)}=3,560\right.$ ecorrelação significante ao nível de0,016), na adoção do comportamento do não uso de bebidas e direção em universitários. 
Também foi realizada uma análise dos testes qui-quadrado, com o objetivo de verificar se realmente havia diferença significativa entre as três condições experimentais de comunicações persuasivas e o grupo de controle em relação à intenção comportamental em adotar o comportamento do não ingerir álcool e direção, ou seja, a variável dependente (VD). A análise do testes qui-quadrado também revelou haver diferenças significativas entre as três condições experimentais de comunicações persuasivas e o grupo de controle $\left({ }^{2}=12,71\right.$, e correlação significante ao nível de $p<0,005)$. Análises posteriores entre as várias médias indicaram haver diferenças significativas entre o GE1 e GSC e entre GE2 e GSC e entre GCP e GSC.

Com alusão ao teste experimental das comunicações persuasivas e a hipóteses experimentais, os resultados apresentados pela análise dos testes qui-quadrado corroboraram parcialmente e permitiram concluir que:

A hipótese específica 1 assegurava que o grupo submetido à comunicação persuasiva positiva explicaria maior variância da intenção comportamental que os grupos submetidos à comunicação persuasiva negativa, contudo, não foi estatisticamente significativa não confirmando essa hipótese. Em relação às hipóteses específicas 2 e 3, também não foram estatisticamente significativas.

Atendendo ao segundo objetivo específico desta investigação e à quarta hipótese alternativa específica associada, rejeitou-se a hipótese de nulidade, dado que as correlações (simples e múltiplas) e as regressões múltiplas demonstraram que as quatro condições estudadas (conforme a Tabela 2) permitiram corroborar empiricamente a validade teórica e metodológica da Teoria da Ação Racional de Ajzen e Fishbein (1980) e Fishbein e Ajzen (1975).

As análises estatísticas efetuadas para cada uma das condições experimentais revelaram que as comunicações persuasivas surtiram o efeito desejado, qual seja o de sensibilizar as intenções comportamentais dos estudantes universitários na direção da adoção do comportamento de prevenção, para variável intenção comportamental, $\left(F_{(3.162)}=3,560\right.$
eCorrelação significante ao nível dep < 0,016), na adoção do comportamento do não uso de álcool e direção.

Sabe-se que a abordagem cognitivo-comportamental compreende o uso de substâncias psicoativas e sua consequente dependência como um comportamento adquirido por meio de formas de aprendizagem, tais como imitação de pessoas importantes, e que teorias do desenvolvimento psicológico enfatizam os pares como influentes nos hábitos dos jovens. A influência dos referentes na intenção de consumo de álcool, por exemplo, foi demonstrada entre adolescentes de $\mathrm{Mi}$ chigan, EUA (Marcoux \& Shope, 1997). De forma semelhante, no presente estudo, os jovens também consideraram a opinião dos referentes como significativas quanto à intenção de não consumir bebidas e direção. Para esses universitários, a intenção de não usar bebidas alcoólicas e direção depende da opinião dos referentes (pais, irmãos, pares e profissionais de saúde).

A questão da influência dos referentes sobre o hábito de não usar bebidas também foi investigada por Bezerra (1997) quase que de forma semelhante ao presente estudo. O autor detectou uma influência marcante dos referentes na intenção comportamental de não ingerir bebidas alcoólicas em jovens universitários. Assim, no estudo de Bezerra (1997) e na presente investigação, abordase a influência dos referentes sobre o não consumo de álcool, percebe-se uma convergência entre os resultados: para o autor, os jovens consideram a opinião dos referentes significativa quanto à intenção de não ingerir bebidas. No presente estudo, também se observou que os referentes afetam a intenção do não consumo de álcool e direção pelos universitários.

Tem-se que essa convergência dos resultados resulte da mesma intenção investigada. No estudo de Bezerra (1997), a intenção era a de não usar álcool, enquanto na presente investigação também a intenção é de não usar bebidas alcoólicas só que acrescentando o ato de dirigir. Isso significa dizer que, para os jovens, a opinião dos referentes é preditora do comportamento, no caso de não uso de 
álcool e direção. Esses resultados sugerem que, apesar de investigações constatarem um estímulo ao consumo na nossa sociedade por parte dos pais e dos amigos (CRATOD, 2009), não parece ser esse um motivo direto que leve os universitários a terem intenção de consumir etílicos, sendo necessário, dessa forma, investigar novas influências, como a presença persuasiva da mídia na vida dos jovens universitários.

Tem-se assim que o impacto da propaganda de bebidas sobre o hábito de consumir álcool (ou quaisquer outros produtos que possam gerar dependência) ainda é pouco investigado no Brasil. Levantamentos atuais realizados nos Estados Unidos vêm mostrando que adolescentes possuem extraordinária memorização de propagandas de alimentos poucos saudáveis, álcool e cigarro (Collins, Schell, Ellickson, \& McCaffrey, 2003). Para Unger et al. (1995, citado por Collins et al., 2003), mostraram também que mais da metade dos adolescentes entrevistados em uma pesquisa no ensino médio recordaram uma propaganda de uma determinada cerveja. Já para Aitken et al. (1988) citado por Collins et al., (2003), em estudo com adolescentes, também nos EUA, constataram que $83 \%$ dos adolescentes reconheceram pelo menos a metade das propagandas de bebidas alcoólicas mostradas.

De modo geral, os resultados encontrados no presente estudo, para o comportamento do não consumo etílico e direção, foram satisfatórios e corresponderam ao que é proposto pela TAR e pelo modelo da abordagem da comunicação persuasiva alternativa de Ajzen e Fishbein (1980) e Fishbein e Ajzen (1975).

\section{Considerações finais}

As investigações que procuram estudar o comportamento mostram, em um sentido amplo, determinados entraves, sendo um deles a própria forma como os dados são obtidos. Assim sendo, não se pode deixar de levar em conta que os questionários são instrumentos fortes de coleta de dados e a não identificação do respondente auxilia a tornar as respostas mais seguras. Também há outras vantagens investigativas do uso do questionário, como, por exemplo: a autoobservação dos processos mentais, evitando-se especulações acerca de estados interiores; resgate de estados mentais via memória; observação do significado e motivações subjacentes ao comportamento; e, finalmente, a facilidade e presteza na coleta de dados para tratamento estatístico.

Deve-se também considerar que a avaliação de tais estratégias precisa ser realizada, sistematicamente, por meio de um planejamento rigoroso, preferencialmente do tipo antes-edepois. Não obstante isso, a necessidade do suporte de um referencial teórico refutável (comumente negligenciado nas comunicações massivas) é condição fundamental para se proceder de forma parcimoniosa e inteligível as devidas explicações pelo fracasso (ou mesmo, aparente sucesso) das campanhas institucionais de incentivo não ingerir álcool quando for dirigir.

É importante também frisar que os motivos pelos quais os universitários são levados ao não uso e uso de etílico são bem diversificados, ainda mais quando se levam em conta os diversos meios culturais. Dificilmente a complexidade das razões de cada jovem ou indivíduo seria mensurável em um estudo desta magnitude, bem como as influências que ele recebe do meio. Mesmo assim, pode-se afirmar que, por se tratar da própria investigação desta dimensão em uma cidade como o Recife, os resultados são satisfatórios.

Assim, é essencial discutir que a TAR tem metodologia própria e relevante por propor a elaboração de um instrumento, neste caso, o questionário utilizado no estudo experimental das Comunicações Persuasivas, não partindo de forma casual de crenças encontradas na literatura. No entanto, esse método de levantamento pode em algumas situações, restringir as variáveis que influenciam o não uso de álcool pelo jovem, o que pode provocar déficit nos resultados.

Por exemplo, temos conhecimento do enorme papel da mídia no que se refere ao alcance 
na propagação do uso de bebidas alcoólicas. No entanto, pela metodologia adotada, tal componente não pode ser pesquisado, uma vez que não foi referido pelos universitários, até mesmo pelo fato de não haver questão abordando outras variáveis que influenciam o consumo, afora aquelas ligadas aos referentes, que formam a norma subjetiva. Ressaltamos, dessa forma, que o ponto referente aos processos midiáticos necessita ser adicionado em outras investigações que envolvam o tema e a amostra aqui estudada.

\section{Nemésio Dario Almeida}

Doutor em Psicologia, Analista Judiciário, Psicólogo do TJ-PE, Pesquisador do Grupo do Núcleo de Pesquisa em Epistemologia Experimental e Cultural - NEC do PPG em Psicologia Cognitiva da UFPE, Recife - PE - Brasil. Email: nemesiodario@hotmail.com

\section{Antonio Roazzi}

D.Phil Oxford, Professor Titular, Depto Psicologia e PPG em Psicologia Cognitiva, Coordenador do Núcleo de Pesquisa em Epistemologia Experimental e Cultural (NEC), Universidade Federal de Pernambuco, Programa de Pós-Graduação em Psicologia Cognitiva, Recife - PE - Brasil. E-mail: roazzi@gmail.com

\section{Endereço para envio de correspondência:}

Rua Adonis de Souza no26-Qd.19, Pina, CEP 51110-210, Recife, PE, Brasil. E-mail: nemesiodario@hotmail.com Fone: (81) 30335809

Recebido 22/03/2013, 1a Reformulação 28/06/2013, Aprovado 29/08/2013. 


\section{Referências}

Ajzen, I., \& Fishbein, M. (1980). Understanding attitudes and predicting social behavior. Englewood Cliffs, New Jersey: Prentice-Hall.

Ajzen, I., Albarracín, D., \& Hornik, R. (2007). Prediction and change of health behavior:Applying the reasoned action approach. Mahwah, NJ: Lawrence Erlbaum Associates.

Almeida, N. D. V. (2003). Circulação humana e subjetividade. In A. C. R. Tupinambá (Org.), Estudos de Psicologia do Trânsito (pp.89-101). Fortaleza, CE: UFCE-Expressão Gráfica.

Almeida, N. D. V. (2006). Promoção e divulgação de medidas educativas em circulação humana: Em questão o fenômeno trânsito brasileiro. Psicologia Argumento, 24(46), 45-53.

Almeida, N. D. A. (2010a). A ingestão de álcool e direção no contexto universitário, comunicação persuasiva e prevenção: uma aplicação da Teoria da Ação Racional. Tese de Doutorado, Programa de Pós-Graduação em Psicologia Cognitiva, Universidade Federal de Pernambuco, PE.

Almeida, N. D. A. (2010b). Considerações acerca da incidência do estresse em motoristas profissionais. Revista de Psicologia, Fortaleza, 1(1), 75-84. Recuperado de http://www.revistapsicologia.ufc.br/images/pdf/ano1edicao1/a no1edicao1006.pdf

Almeida, N. D. V. (2011). Uso de álcool, tabaco e drogas por jovens e adultos da cidade do Recife. Psicologia Argumento, 29(66), 295-302.

Almeida, N. D. V. (2013). A saúde no Brasil, impasses e desafios enfrentados pelo Sistema Único de Saúde-SUS. Revista Psicologia e Saúde, 5(1), 01-09. Recuperado de http://pepsic.bvsalud.org/scielo.php?script $=$ sci_arttext \&pi $\mathrm{d}=$ S 2177-093X2013000100002 \&lng $=\mathrm{pt} \& \mathrm{nrm}=\mathrm{iso} \& \operatorname{lng}=\mathrm{pt}$

Almeida, N. D. V., Dias, M. R., Souza, A. M., Cordeiro, A. M., \& Chaves, S. M. (2004). O consumo de bebidas alcoólicas na condução de veículos: Um estudo de crenças. Revista Symposium, 8(1), 24-37.

Almeida, N. D. V., \& Roazzi, A. (no prelo). Álcool e direção: prevenção em estudantes universitários, uma aplicação da Teoria da Ação Racional. Psicologia: Teoria e Pesquisa, UnB.
Bastos, I. (2000). Intenção do tabagismo pós-conhecimento do enfisema pulmonar. Dissertação de Mestrado em Psicologia Social, Universidade Gama Filho, Rio de Janeiro.

Bezerra, A. E. (1997). Bebidas alcoólicas no contexto universitário: investigação fundamentada na Teoria da Ação Racional. Dissertação de Mestrado, Programa de Pós-Graduação em Psicologia Social, Universidade Federal da Paraíba, Paraíba.

Bisquerra, R., Sarriera, Jorge C. \& Martínez, F. (2007). Introdução à estatística: enfoque informático com o pacote estatístico SPSS. Porto Alegre: Artmed.

Brasil. (2008). Lei $n^{0} 11.705$, de 19/06/2008, dispõem sobre a inibição do consumo de bebida alcoólica por condutor de veículos automotor, e dar outras providências. Recuperado dehttp://www.planalto.gov.br/ccivil_03/_Ato200 7-2010/2008/Lei/L11705.htm.

Breinbauer, C.,\& Maddaleno, M. (2008a). Teoria da Ação Racional. In C. Breinbauer,\& M. Maddaleno (Orgs.), Jovens: escolhas e mudanças, promovendo comportamentos saudáveis em adolescentes. (Mônica G. Armando, trad., pp. 58-63). São Paulo: Roca.

Breinbauer, C.,\& Maddaleno, M. (2008b). Teoria do Comportamento Planejado. In C. Breinbauer \&, M. Maddaleno (Orgs.), Jovens: escolhas e mudanças, promovendo comportamentos saudáveis em adolescentes. (Mônica G. Armando, trad., pp. 64-70). São Paulo: Roca.

Campbell, D. T., \& Stanley, J. C. (1979). Delineamentos experimentais e quase-experimentais de pesquisa. São Paulo: Editora da Universidade de São Paulo.

Cavalcanti, A. P. R., Dias, M. R., Rodrigues, C. F. F., Gouveia, C. N. N. A., Ramos, D. D., \& Serrano, F. J. O. (2007). Crenças e influências sobre dietas de emagrecimento entre obesos de baixa renda. Ciência \& Saúde Coletiva, 12(6), 65-76. doi: 10.1590/S141381232007000600018

Centro de Referência em Álcool, Tabaco e Outras Drogas -CRATOD (2009). Levantamento sobre o uso de drogas em adolescentes brasileiros. Recuperado de http://www.cratod.sp.org.br 
Collins, R., Schell, T., Ellickson, P. L., \& McCaffrey, D. (2003). Predictors of beer advertising awareness among eighth graders. Addction, 98(9), 1297-1306. doi: 10.1046/j.13600443.2003.00448.x

Conselho Federal de Psicologia. (2010). Subjetividade do consumo de álcool e outras drogas e as políticas públicas. Recuperado de http://site.cfp.org.br/publicacao/subjetividade-do-consumo-de-lcool-e-outras-drogas-eas-polticas-pblicas-brasileiras.

Cunha, B. G. F., \& Dias M. R. (2008). Comunicações persuasivas e doação regular de sangue: um estudo experimental. Cad. Saúde Pública, 24(6), 1407-1418. doi: http://dx.doi.org/10.1590/S0102 311X2008000600021

Cozby, P. C. (2009). Métodos de pesquisa em ciências do comportamento. São Paulo: Atlas.

D'Amorim, M. A. (1996). A medida da Teoria da Ação Racional. In: L. Pasquali (Org.), Teoria e métodos de medida em ciências do comportamento (pp. 98-112). Brasília, DF: Instituto Nacional de Estudos e Pesquisas Educacionais.

D’Amorim, M. A. (2004). Teoria da Ação Racional. In M. F. Dela Coleta (Org.), Modelos para pesquisa e modificação de comportamento de saúde. (pp. 63-88). Taubaté, SP: Cabral Editora e Livraria Universitária.

D'Amorim. M. A.,\& Vera, V. M. L. M. (1996). A relação atitude/comportamento à luz da Teoria da Ação Racional. Arquivos Brasileiros de Psicologia, 38(1), 133-42.

Dela Coleta, M. F. (2004).Pesquisa e modificação de comportamento de saúde. Taubaté, SP: Cabral Editora e Livraria Universitária.

Dias M. R. (1995). Aids, comunicação persuasiva e prevenção: uma aplicação da teoria da ação racional. Tese de Doutorado, Programa de Pós-Graduação em Psicologia Social, Universidade de Brasília, Brasília, DF.

Dias, M. R., \& Cavalcanti, A. P. R. (2005). Psicologia e nutrição: predizendo a intenção comportamental de aderir a dietas de redução de peso entre obesos de baixa renda. Estudos de Psicologia(Natal), 10(1), 121129. doi:http://dx.doi.org/10.1590/S1413294X2005000100014
Duailibi, S., Pinsky, I., \& Laranjeira, R. (2010). Álcool e direção: beber ou dirigir: um guia prático para educadores, profissionais da saúde e gestores de políticas públicas. São Paulo: Unifesp.

Fishbein, M., \& Ajzen, I. (1975). Belief, attitude, intention and behavior: An introduction to theory and research. Reading, Massachusetts: Addison-Wesley Publishing.

Fishbein, M., \& Ajzen, I. (2010). Predicting and changing behavior: The reasoned action approach. New York: Psychology Press.

Formiga, A. C. (2002). A prática da caminhada na prevenção do infarto: uma investigação baseada na Teoria da Ação Racional. Dissertação de Mestrado, Programa de Pós-Graduação em Psicologia Social, Universidade Federal da Paraíba, Paraíba.

Formiga, A. S. C., Dias, M. R.,\& Saldanha, A. A. W. (2005). Aspectos psicossociais da prevenção do infarto: construção e validação de um instrumento de medida. PsicoUSF, 10 (1), 31-40. doi: $\quad$ http://dx.doi.org/10.1590/S141382712005000100005

Guimarães, P. V. (2004). Uso de bebidas alcoólicas na adolescência: Investigação fundamentada na Teoria da Ação Racional (TAR). Dissertação de Mestrado, Programa de Pós-Graduação em Psicologia Cognitiva, Universidade Federal de Pernambuco, Pernambuco.

Günther, H. (2003). [Auto]-avaliação de um relato ou projeto de pesquisa. Brasília, DF: Laboratório de Psicologia Ambiental, Universidade de Brasília. (Série Planejamento de Pesquisa nas Ciências Sociais, 6).

Instituto de Pesquisa Econômica Aplicada. (2003). Impacto social e econômico dos acidentes de trânsito nas aglomerações urbanas brasileirasSíntese da pesquisa. Recuperado de http://www.ipea.gov.br.

Instituto de Pesquisa Econômica Aplicada. (2006). Impactos sociais e econômicos dos acidentes de trânsito nas rodovias brasileiras. Relatório Executivo. Recuperado de http://www.ipea.gov.br.

Maia, T. P. A. (2009). Vítimas de acidentes rodoviários. In A. S. Bianchi (Org.), Humanidade e trânsito: desafios para um futuro sustentável (pp.165-184). Curitiba, PR: Conselho Regional de Psicologia da 8a Região. 
Marcoux, B. C., \& Shope, T. J. (1997). Application of the theory of planned behavior to adolescent use and misuse of alcohol. Health Education Research, 12(3), 323-331.doi: 10.1080/08870440902777554.

Mendes, A. C. G., Sá, D. A., Miranda, G. M. D., Lyra, T. M.,\& Tavares, R. A. W. (2012). Assistência pública de saúde no contexto da transição demográfica brasileira: exigências atuais e futuras. Caderno de Saúde Pública, 28(5), 955-964. doi: http://dx.doi.org/10.1590/ S0102-311X2012000500014

Michener, H. A., Delamate, J. D., \& Myers, D. J. (2005). O modelo da ação racional. In H. A. Micheber, J. D. Delamate, \& D. J. Myers (Orgs.), Psicologia social (pp.199-201). São Paulo: Thomson Pioneira.

Ministério da Saúde. (2012). Sistema de Informações sobre Mortalidade-SIM-DATASUS. Morbidade por acidentes e violências no Brasil: tendência das hospitalizações no período de 2002 a 2011. Recuperado de http://portalsaude.saude.gov.br/portalsaude/arquivos/pdf/20 13/Fev/21/saudebrasil2011 parte1_cap9.pdf.

Moutinho, K. (2000). Amamentação à luz da Teoria da Ação Racional: Crenças, normas, atitudes e intenções. Dissertação de Mestrado, Programa de Pós-Graduação em Psicologia Cognitiva, Universidade Federal de Pernambuco, Pernambuco.
Moutinho, K., \& Roazzi, A. (2010). As teorias da ação racional e da ação planejada: relações entre intenções e comportamentos. Avaliação Psicológica, 9(2), 279-287. Recuperado de http://pepsic.bvsalud.org/scielo.php?script=sci arttext\&pid $=$ S1677-04712010000200012

Ramos-Álvarez, M. M., \& Catena, A. (2004). Normas para elaboración y revisión de artículos originales experimentales en ciencias del comportamiento. International journal of clinical and health psychology, 4(1), 173-89.

Santanna, M. (2005). Doação de sangue voluntária e periódica: uma aplicação da Teoria da Ação Racional. Dissertação de Mestrado, Programa de Pós-Graduação em Psicologia Social, Universidade Gama Filho, Rio de Janeiro.

Thielen, I. P.,\& Soares, D. P. (2009). Percepção de riscos: sexo, drogas e... (Rock'n'roll) trânsito. In A. S. Bianchi (Org.), Humanidade e trânsito: desafios para um futuro sustentável (pp.133146). Curitiba, PR: Conselho Regional de Psicologia da $8^{\underline{a}}$ Região.

Tawil, M. (2007). Trânsito assassino. São Paulo: Albatroz.

World Health Organization (WHO). (2011) Global Status Report on Road Safety: Supporting a decade of action. Geneva. Recuperado de http://www.who.int. 